\title{
Numerical and experimental studies of cavitation behavior in water-jet cavitation peening processing
}

\author{
H. Zhang ${ }^{\mathrm{a}}$, B. Han ${ }^{\mathrm{a}, *}$, X.G. Yu ${ }^{\mathrm{a}}$ and D.Y. Ju ${ }^{\mathrm{b}}$ \\ ${ }^{a}$ School of Mechanical Engineering and Automation, University of Science and Technology Liaoning, Anshan, \\ Liaoning, China \\ ${ }^{\mathrm{b}}$ Department of Material Science and Engineering, Saitama Institute of Technology, Fukaya, Saitama, Japan
}

Received 17 July 2012

Revised 22 February 2013

Accepted 25 March 2013

\begin{abstract}
Water-jet cavitation peening (WCP) is a new technology for the surface modification of metallic materials. The cavitation behavior in this process involves complex and changeable physics phenomena, such as high speed, high pressure, multiple phases, phase transition, turbulence, and unstable features. Thus, the cavitation behavior and impact-pressure distribution in WCP have always been key problems in this field. Numerous factors affect the occurrence of cavitation. These factors include flowboundary conditions, absolute pressure, flow velocity, flow viscosity, surface tension, and so on. Among these factors, pressure and vapor fraction are the most significant. Numerical simulations are performed to determine the flow-field characteristics of both inside and outside the cavitating nozzle of a submerged water jet. The factors that influence the cavitation intensity of pressure are simulated. Fujifilm pressure-sensitive paper is used to measure the distribution of impact pressure along the jet direction during the WCP process. The results show that submerged cavitation jets can induce cavitation both inside and outside a conical nozzle and a convergent-divergent nozzle when the inlet pressure is $32 \mathrm{MPa}$. Moreover, the shock wave pressure induced by the collapse of the bubble group reaches up to $300 \mathrm{MPa}$.
\end{abstract}

Keywords: Water-jet cavitation peening, numerical simulation, pressure-sensitive paper, impact wave pressure

\section{Introduction}

Water-jet cavitation peening (WCP) is a novel surface-strengthening technology. A large number of small bubble clouds that are generated by a submerged cavitation jet with high velocity and pressure collapse on the surface of a metallic material. This process can produce impact pressure of up to several GPa and can be used to strengthen metallic materials [1-3]. Recent developments showed that this new technology can produce a compressive residual stress layer near the surface of metallic parts, similar to that produced by other applications of shot peening technology. This layer improves the fatigue life of the parts [4-6]. However, the cavitation behavior of the aforementioned process includes complex phenomena, such as high speed, high pressure, multiple phases, phase transition, turbulence, and unstable features. Research on the basic theory of cavitation behavior and impact-pressure distribution remains relatively weak. This fact hinders further improvement of WCP.

\footnotetext{
* Corresponding author: Bing Han, School of Mechanical Engineering and Automation, University of Science and Technology Liaoning, No. 185, Qianshan Midroad, Hi-Tech Zone, Anshan 114051, Liaoning, China. Tel.: +86 4125928271 ; Fax: +86 412 592 8271; E-mail: hanb75@126.com.
} 
An increasing demand for the development and validation of the computational fluid dynamics (CFD) of numerical models has emerged in literature. This demand, along with the continuing efforts to obtain better experimental information under the most realistic conditions possible, has given rise to the simulation of the formation and development of cavitation inside a nozzle.

Yu et al. [7] investigated the collapse of a cavitation bubble in a shear flow. The bubble collapse in a quiescent fluid can be solved by the Rayleigh-Plesset equation for bubble dynamics. Yu et al. observed that the collapse rate of a bubble in a shear flow increases if the shear is sufficiently strong. Deshpande et al. [8] developed a solution based on the two-dimensional (2D) Navier-Stokes equations. The energy equation uses artificial compressibility and pseudo-time stepping techniques to model the thermodynamic effects of sheet cavitation in cryogenic fluids, which could be used to examine individual bubbles. Yuan et al. [9] performed a numerical simulation of cavitation phenomena, including the growth and collapse of bubbles inside an injector nozzle, using a $k-\omega$ turbulence model.

This study aims to explain the cavitation phenomenon using different CFD cavitation models. Each model is based on a different assumption. Various numerical methods have been used in studying commercial fluid-flow solvers. Most models are based on the assumption that cavitation is a mechanically driven phenomenon initiated by the presence of cavitation nuclei that grow to become bubbles or bubble clouds, which then form the complex cavitation structures observed experimentally [10]. Srinivasan et al. recently developed a novel modeling approach capable of simultaneously tracking cavitation events occurring within an injector nozzle with the random number generator (RNG) $\kappa-\varepsilon$ turbulence model. This model includes new source terms from cavitation-induced turbulent kinetic energy production and dissipation [11]. Li et al. adopted the volume of fluid model and the unsteady method to simulate the velocity of a micro jet generated by a bubble collapsing near a solid wall. The impact pressure on the solid wall reaches up to $3.0 \times 10^{8} \mathrm{~Pa}$ when the velocity of the micro jet is $200 \mathrm{~m} / \mathrm{s}$ [12]. Deng et al. [13] performed numerical simulations on the cavitation flow in an angle nozzle adopting a $\kappa-\varepsilon$ turbulence model using the SIMPLEC method in the FLUENT software., The validity of the simulation results were verified. Lu et al. [14] investigated the applicability of the standard $\kappa-\varepsilon$, RNG $\kappa-\varepsilon$, and standard $\mathrm{k}-\mathrm{w}$ turbulent models for cavitation in a water jet field through a convergent-divergent nozzle. The result of Lu's study indicated that the RNG $\kappa-\varepsilon$ turbulence model is the most suitable for the simulation of cavitation behavior. Qin et al. [15] established a model based on the RayleighPlesset bubble dynamics equation with a variable time-step technique to solve the highly nonlinear second-order differential equation. This model can simulate the process of growth, as well as the collapse and rebound of a cavitation bubble traveling along the flow through a convergent-divergent nozzle that generates a cavitation jet.

However, reports on cavitation behavior in the WCP process remain limited. In this paper, numerical and experimental studies on the cavitation behavior in WCP were performed. For numerical simulations, FLUENT was used to simulate cavitation behavior both inside and outside the conical nozzle and the convergent-divergent nozzle. The cavitation behavior induced by the convergent-divergent nozzle with different inlet pressure values was also investigated. To validate the process capability of the cavitation jet, Fujifilm pressure-sensitive paper was used to measure the distribution of the impact pressure along the jet direction in the WCP process.

\section{Mathematic model}

\subsection{Governing equations}

$k$ is assumed to be the turbulence kinetic energy, $\varepsilon$ is the turbulence dissipation rate of the unit mass fluid, $u$ is the axis velocity, $v$ is the radial velocity, and $\rho$ is the density. The 2D flow model of the incompressible fluid with the $k-\varepsilon$ turbulence model under an axial symmetrical cylindrical coordinate is given by Eq. (1) [16]:

$$
\frac{1}{r}\left[\frac{\partial}{\partial_{x}}(r \rho u \phi)+\frac{\partial}{\partial_{r}}(r \rho v \phi)\right]=\frac{1}{r}\left[\frac{\partial}{\partial_{x}}\left(r \Gamma_{\phi} \frac{\partial_{\phi}}{\partial_{x}}\right)+\frac{\partial}{\partial_{r}}\left(r \Gamma_{\phi} \frac{\partial_{\phi}}{\partial_{r}}\right)\right]+S_{\phi}
$$

where $\phi$ represents variables $u, v, k$, and $\varepsilon . S_{\phi}$ is the source term of the $\phi$ equation.

\subsection{Solution procedure and boundary conditions}

Appropriate initial and boundary conditions are needed to solve the Navier-Stokes equations and to make the result reliable. Initial and boundary conditions can have significant influence on the results. Therefore, the initial 


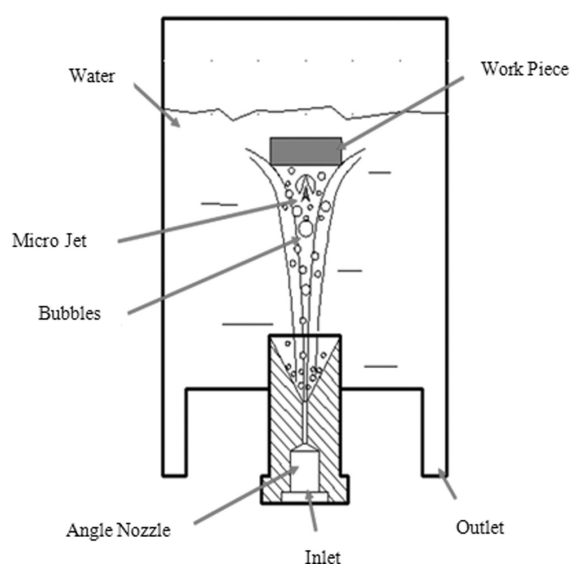

Fig. 1. Schematic of working zone of WCP processing.

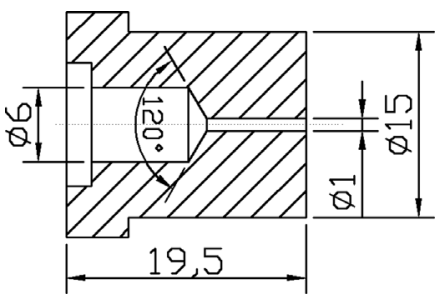

(a) Conical nozzle

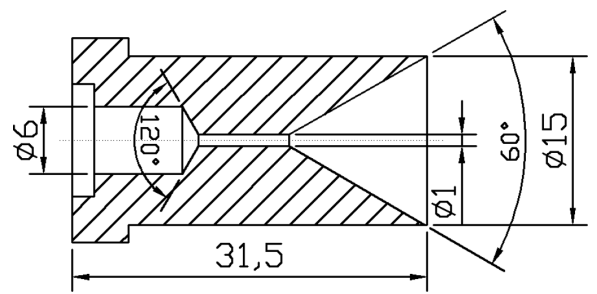

(b) Convergent-divergent nozzle

Fig. 2. Structural scheme of nozzles.

and boundary conditions provided should be as realistic as possible. In this study, a stationary single-phase fluid is assumed to be the initial condition. Both inflow and outflow boundaries are modeled as constant-pressure surfaces. Thus, a converged or near-converged solution for a single-phase liquid flow is obtained. Calculating the cavitation model can easily yield a converged result. For the present computations, the renormalized group RNG $\kappa-\varepsilon$ is adopted with a wall function to calculate the cavitation flow field [17]. The boundary conditions of the wall are impermeability and no-slip for velocity. The normal gradient of pressure is assumed to be zero.

\section{Numerical simulation}

\subsection{Physical modeling and gridding}

During the WCP process, water with a normal temperature is pressurized using a high-pressure piston pump. The pressurized water is jetted by different nozzles in the water tank, thus forming the submerged cavitation jet flow. Large, uniform bubble clouds can then be generated. The bubbles that collapse on the surface of metallic materials will produce a similar effect as that of shot peening. The physical model of the working zone is simplified to simulate cavitation behavior, as shown in Fig. 1. The model has four parts, namely, a high-pressure water inlet, the different nozzles, the atmospheric pressure outlet, and the water-filled working zone. Regarding the symmetry in 2-D axial plane, simplicity and fastness of 2-D approach rather than the 3-D approach, the physical model can be simplified in a 2D axisymmetric model. In this paper, the cavitation behavior induced by the conical nozzle and by the convergent-divergent nozzle is investigated. The structure schemes and sizes of the different nozzles are shown in Fig. 2.

The computational domain and the structured grid system are created using GAMBIT, as shown in Fig. 3. The dimensions of computational domain is $60 \times 30 \mathrm{~mm}^{2}$. The dimensions of atmospheric pressure outlet is $2 \mathrm{~mm}$. The meshed flow domain includes the nozzle and the experimental cavitation cell. The nozzle and the working zone are 


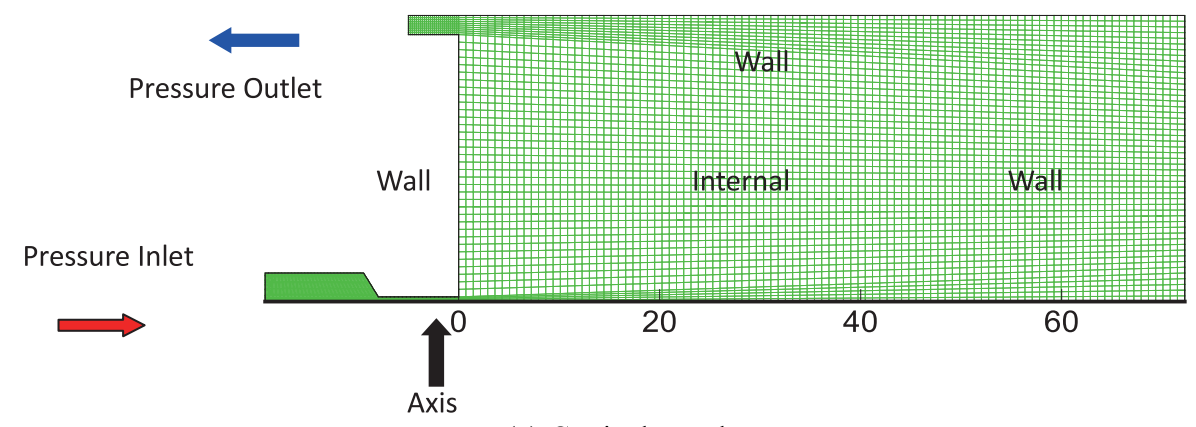

(a) Conical nozzle

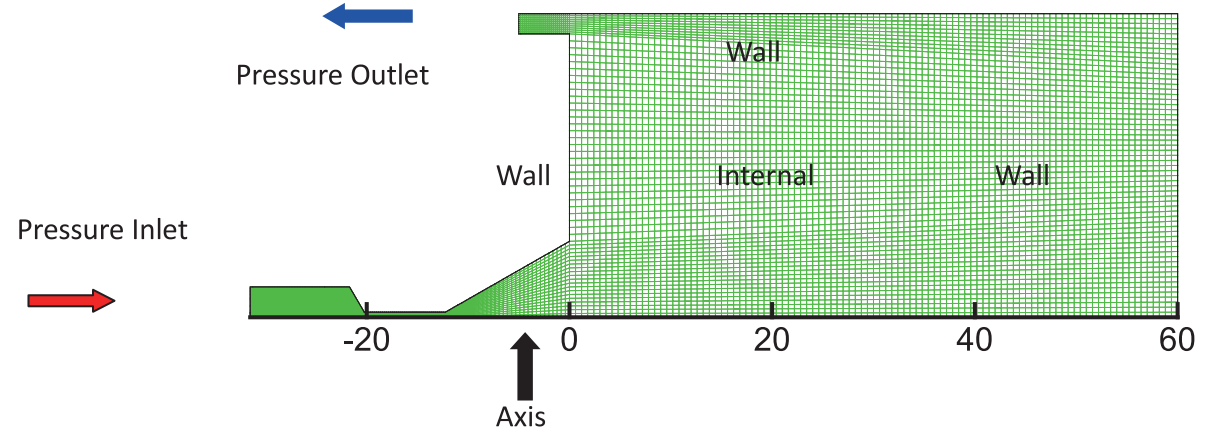

(b) Convergent-divergent nozzle

Fig. 3. Mesh generation of half-flow field.

in axial symmetry. Thus, half of the physical model has to be discretized. The grids have to be locally refined in the areas of nozzle contraction, diffusion, and exit. Governing equations are discretized with a second-order upwind. After the initial analysis, 25804 quad meshes are adequate to meet the requirement in Fig. 3(a). By contrast, 25804 quad meshes are adequate to meet the requirement in Fig. 3(b).

\subsection{Boundary conditions}

The model is simplified according to the process parameters and the actual environmental conditions in the WCP process. The following boundary conditions were applied: (a) The working temperature is $20^{\circ} \mathrm{C}$ during the WCP process. (b) The wall is assumed to be adiabatic and does not occur in the convective heat with the surrounding space. (c) The process is assumed as to be a static problem because the growth, collapse, and rebound of a cavitation bubble traveling along the flow through the nozzles are not considered.

In this paper, the RNG $\kappa-\varepsilon$ turbulence model with standard wall functions was used to simulate the cavitation jet induced by two different nozzles. The inlet boundary condition is the inlet pressure, $32 \mathrm{MPa}$, and the outlet boundary condition is outlet pressure, $101325 \mathrm{~Pa}$. The radial speed of the axial line is $v=0$. The cavitation behavior induced by the conical nozzle and by the convergent-divergent nozzle is simulated. The cavitation behavior induced by the convergent-divergent nozzle with different inlet pressure values $(0.5,2.0,8.0$, and $32.0 \mathrm{MPa})$ is also investigated.

\section{Numerical simulation results}

\subsection{Cavitation behavior with different nozzles}

First, the static pressure of the water jet flow is simulated. The distribution of static pressure along the axis direction with two different nozzles is shown in Fig. 4. The pressure along the jet flow direction gradually drops. The pressure is at its minimum in the throat of the two nozzles. The contraction area for the convergent-divergent 


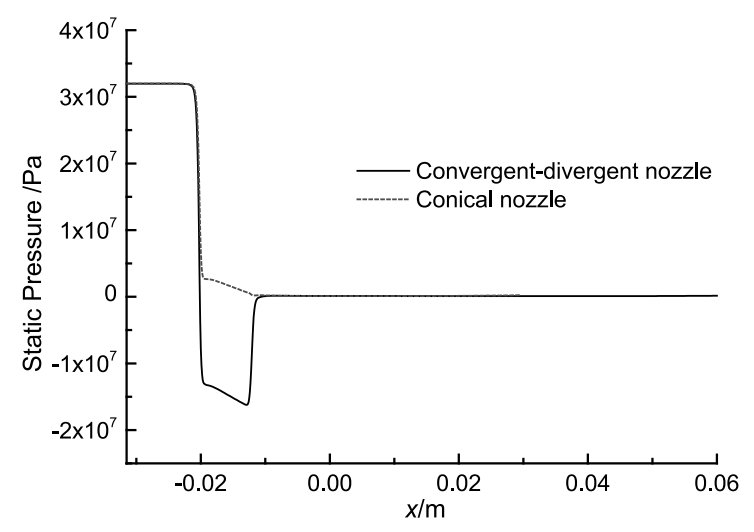

Fig. 4. Distribution of static pressure along the axis direction.
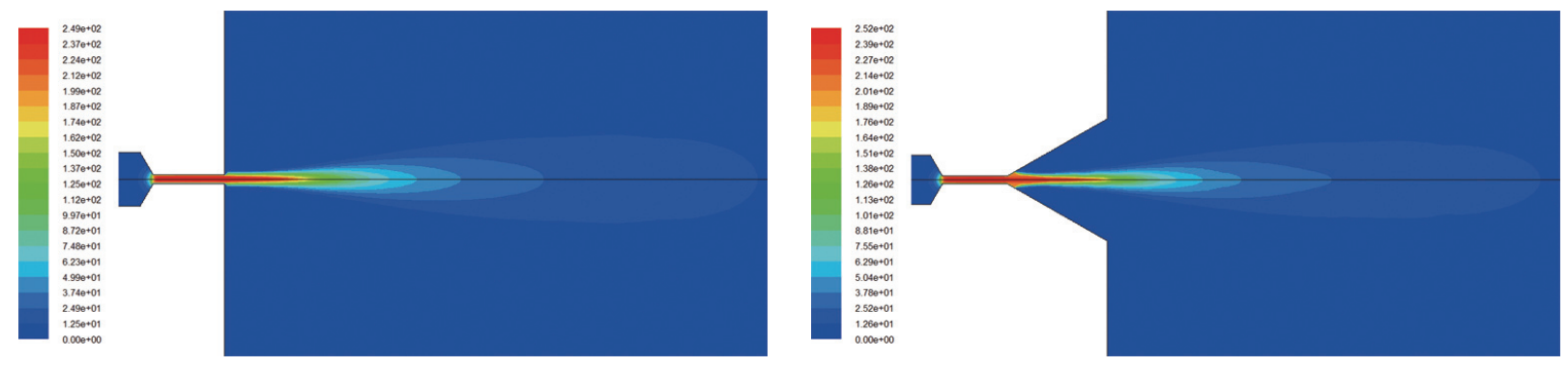

Fig. 5. Distribution of velocity in two different nozzles.
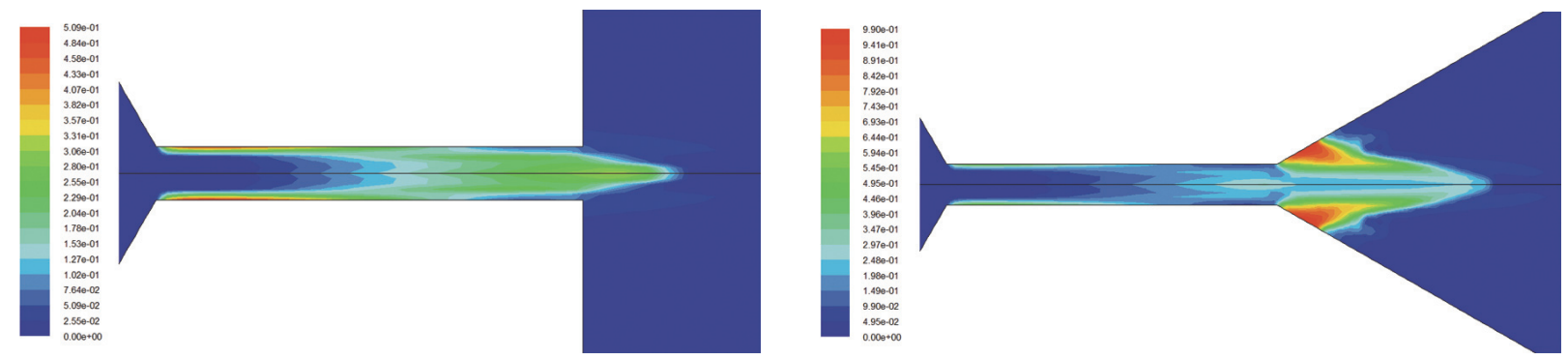

Fig. 6. Distribution of vapor-phase volume.

nozzle can accelerate the drop of pressure, making the static pressure lower than the inlet pressure. The expansion area can reduce speed and increase pressure, thus restoring the flow to its original entry conditions immediately after leaving the outlet. This process also reduces kinetic energy loss. The results show that a pressure drop of $-20 \mathrm{MPa}$ is produced at the throat of the convergent-divergent nozzle, thus promoting the occurrence of cavitation. The pressure drop in the conical nozzle is considerably less than that in the convergent-divergent nozzle. These results indicate that the conical nozzle is unsuitable for water-jet cavitation.

The distribution of the flow velocity field is shown in Fig. 5. A potential core region in the flow field is found in the outlet zones of the two nozzles. The velocity is quickly reduced at the end of the potential core region. Moreover, an obvious change in the velocity gradient normal to the direction of the jet flow is also observed.

The distribution of vapor-phase volume in two different nozzles is shown in Fig. 6. An obvious cavitation phenomenon occurred in the two nozzles. However, the intensity of the cavitation phenomenon in the convergentdivergent nozzle is significantly greater than that in the conical nozzle. The maximum vapor fraction is 0.990 in the convergent-divergent nozzle and 0.509 in the conical nozzle. 


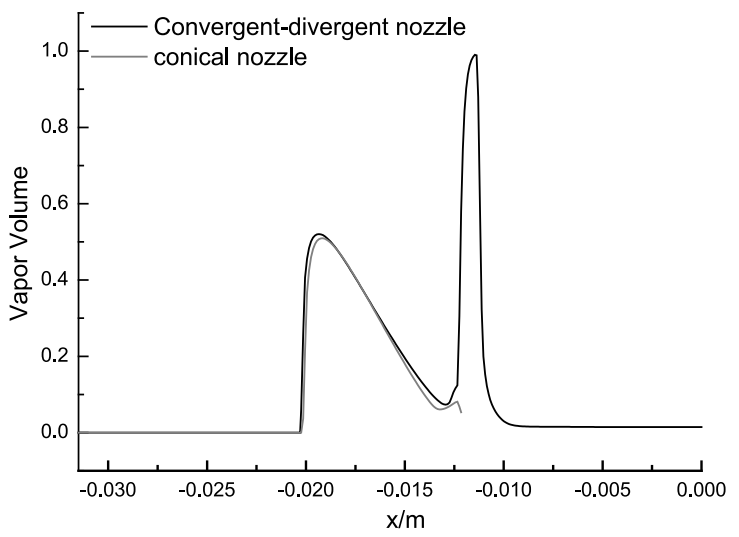

Fig. 7. Distribution of vapor-phase volume along the wall in two different nozzles.
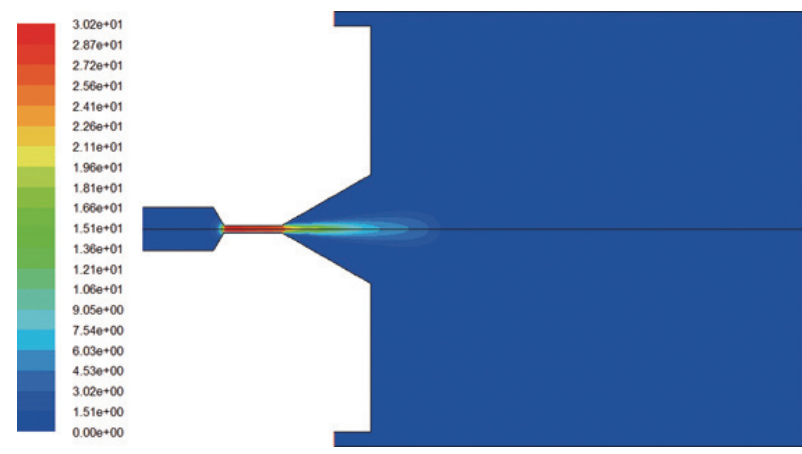

(a) $0.5 \mathrm{MPa}$
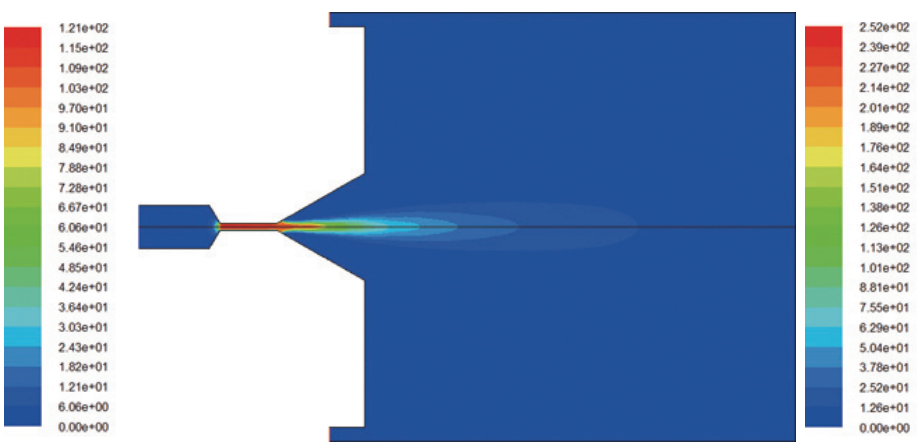

(c) $8 \mathrm{MPa}$

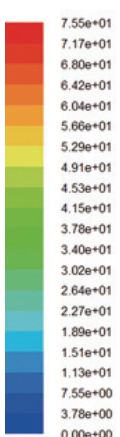

$3.780+00$

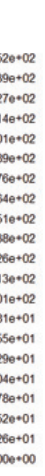

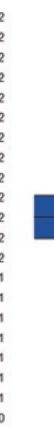

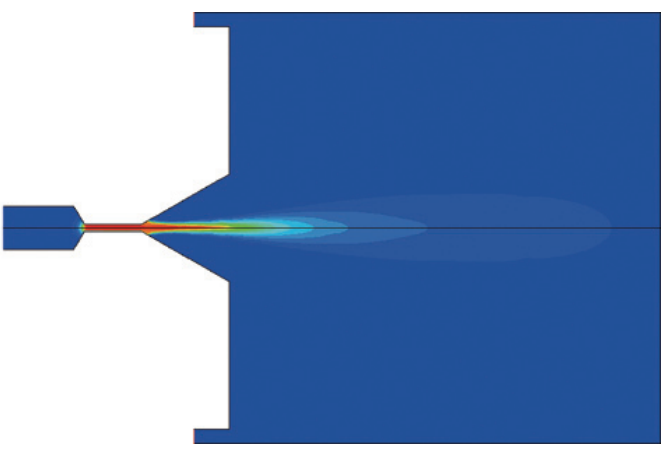

(d) $32 \mathrm{MPa}$

Fig. 8. Distribution of velocity of cavitation field under different inlet pressure values.

The distribution of vapor-phase volume along the wall in two different nozzles is shown in Fig. 7. Two peaks induced by the two corners in the nozzle are found in the convergent-divergent nozzle. The vapor fraction in this region is larger than that in other areas. The maximum vapor fraction is found in the expansion area. In addition, the sudden increase and decrease in vapor fraction respectively represent the growth and collapse of bubbles in the convergent-divergent nozzle.

\subsection{Cavitation behavior with different inlet pressure values}

The RNG $\kappa-\varepsilon$ turbulence model with standard wall functions was used to simulate the cavitation jet in the convergent-divergent nozzle with different inlet pressure values. The inlet pressure values are $0.5,2.0,8.0$, and 


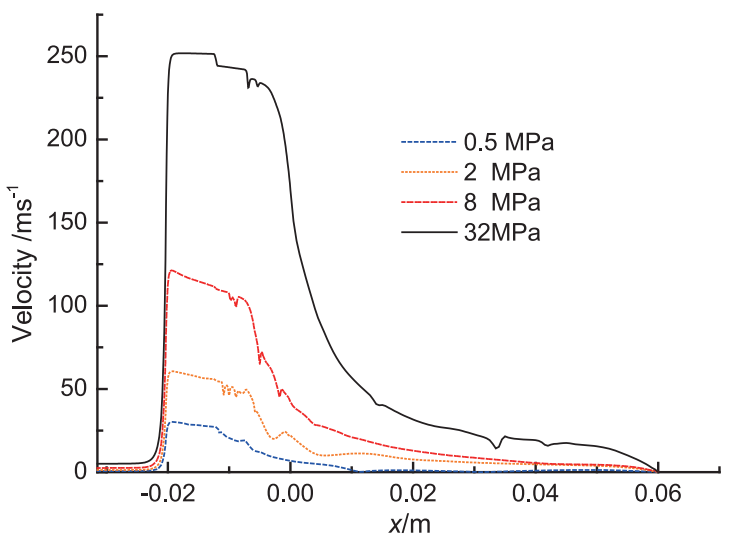

Fig. 9. Distribution of velocity along the $\mathrm{X}$-axis direction under different inlet pressure values.

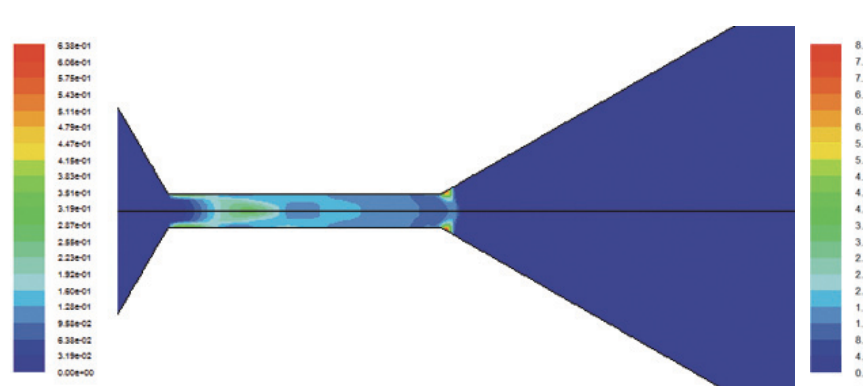

(a) $0.5 \mathrm{MPa}$

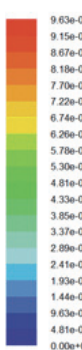

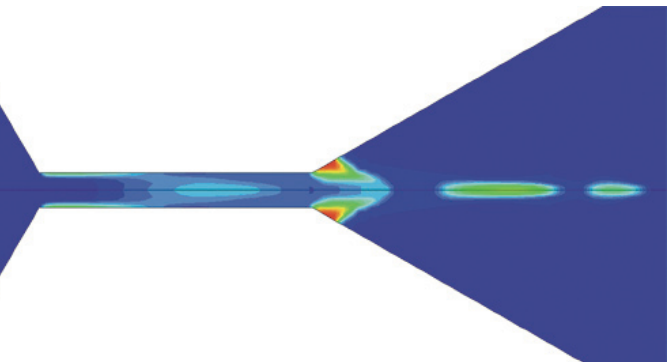

(c) $8 \mathrm{MPa}$

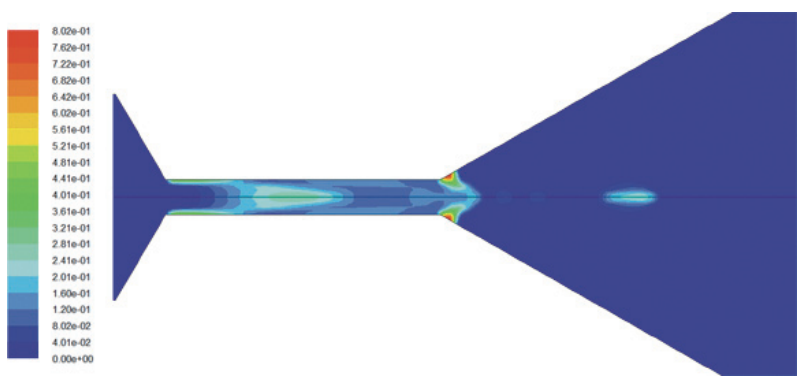

(b) $2 \mathrm{MPa}$

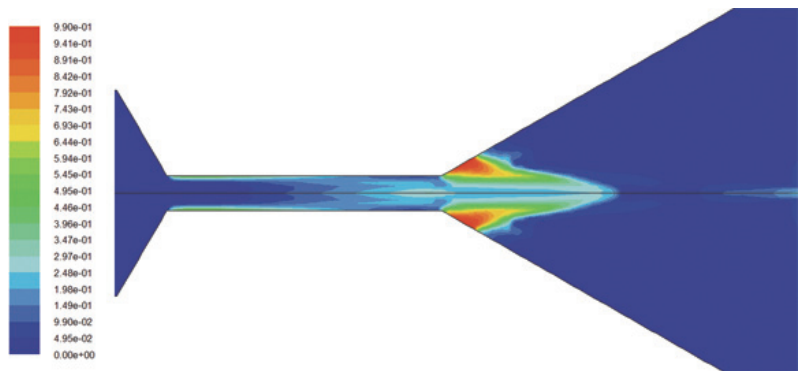

(d) $32 \mathrm{MPa}$

Fig. 10. Distribution of the vapor-phase volume fraction under different inlet pressure values.

32.0 MPa. The outlet pressure is constant at $101325 \mathrm{~Pa}$. The axial boundary condition is $v=0$. The temperature of the working liquid is $20^{\circ} \mathrm{C}$. The distribution of flow-field velocity with different inlet pressure values is shown in Fig. 8. A large flow velocity gradient is found in the nozzle. The velocity along the axis direction obviously increases with increasing inlet pressure, as shown in Fig. 9.

The distribution of the vapor-phase volume fraction in the convergent-divergent nozzle is shown in Fig. 10. The maximum vapor-phase volume fraction is 0.990 at an inlet pressure of $32 \mathrm{MPa}$. On the other hand, the minimum vapor-phase volume fraction is 0.683 at an inlet pressure of $0.5 \mathrm{MPa}$.

The distribution of vapor-phase volume along the wall under different inlet pressure values is shown in Fig. 11. The vapor-phase volume fraction becomes larger with increasing inlet pressure. This finding indicates that increasing inlet pressure can promote the intensity of the cavitation phenomenon with unchanged outlet pressure.

The relationship among inlet pressure, cavitation number, and vapor fraction is shown in Fig. 12. Simulation results show that the maximum vapor-phase volume fraction becomes larger with increasing inlet pressure. Meanwhile, a decrease in cavitation number corresponds with increasing inlet pressure. This result conforms to the experimental result that increasing inlet pressure can promote the intensity of cavitation. This finding indicates that the 


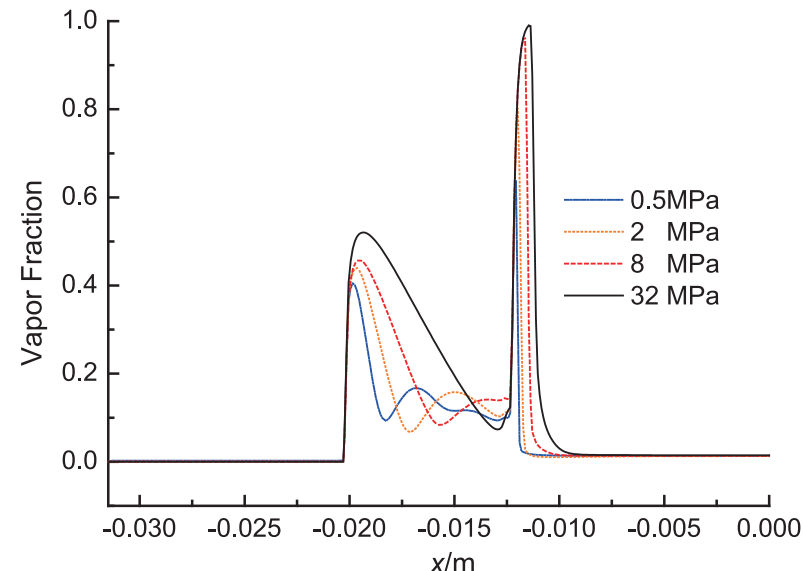

Fig. 11. Distribution of the vapor-phase volume fraction along the wall under different inlet pressure values.

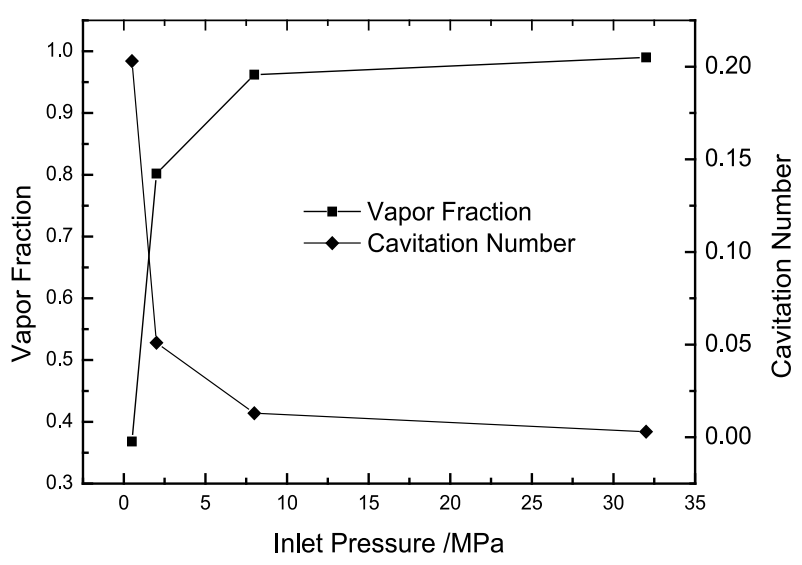

Fig. 12. Relationship among inlet pressure, cavitation number, and vapor fraction.

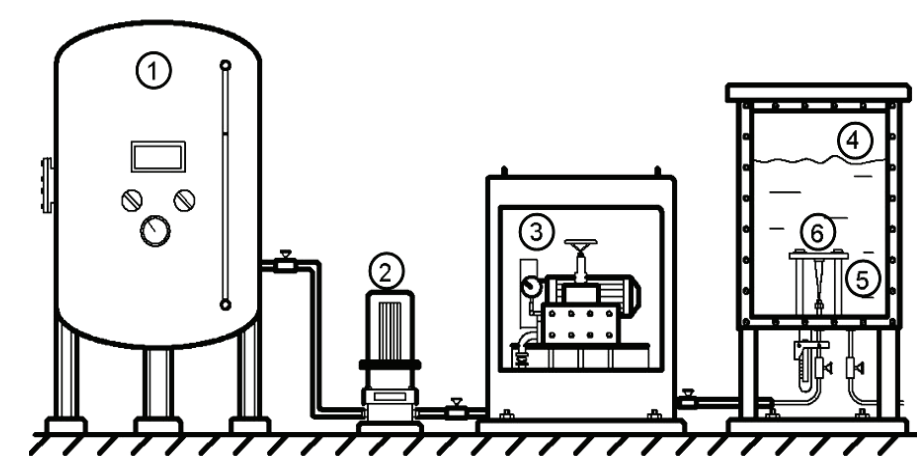

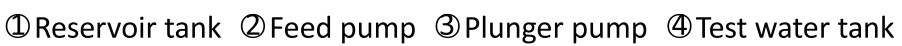

(5) Jet nozzle (b) Test specimen

Fig. 13. Schematic of the WCP device.

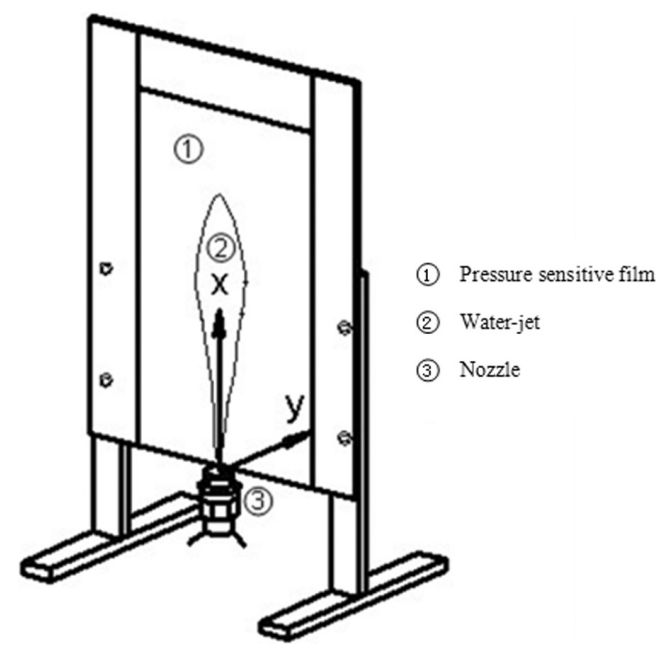

Fig. 14. Schematic of the impact-pressure measuring equipment.

simulation results of the cavitation flow field in the convergent-divergent nozzle with RNG $\kappa-\varepsilon$ turbulence model are reliable.

\section{Experimental studies}

\subsection{Structure of WCP and processing parameters}

The subject structure of the WCP device is illustrated in Fig. 13. This device has five parts, namely, a reservoir tank, a feed pump, a plunger pump, a test-water tank, and a jet nozzle. The working principle is that water is supplied by a pump to the water jet from the reservoir tank. Water goes through a high-pressure pump (the maximum pressure and the flux of the plunger pump are $32 \mathrm{MPa}$ and $16 \mathrm{~L} / \mathrm{min}$, respectively) after pressure from the nozzle drops. The temperature of the working water is maintained at $293 \mathrm{~K}$ to $297 \mathrm{~K}$, forming a vertical submerged cavitation water jet. Micro bubbles can be generated during the WCP process. The bubbles that collapse on the surface of the specimen will produce a huge shock wave pressure. This pressure is used to induce the formation of a compressive residual stress layer on the near surface of the metal parts, thus improving fatigue strength and wear resistance, etc. 


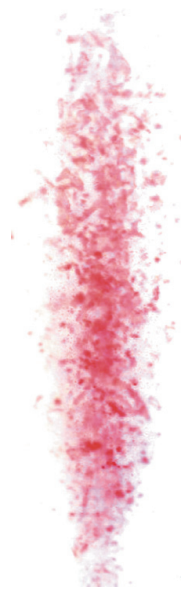

(a) Pressure-sensitive film treated by WCP

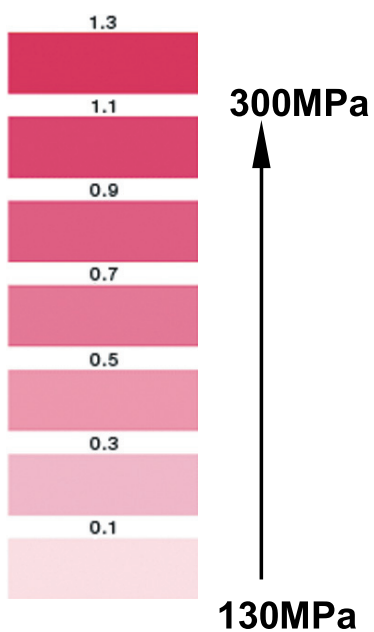

(b) Standard density card

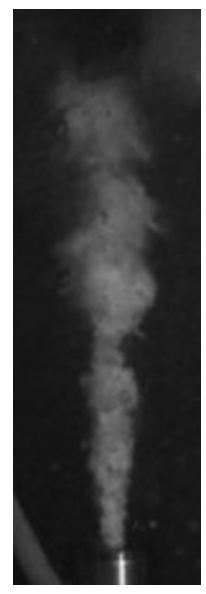

(c) Photo of bubble clouds

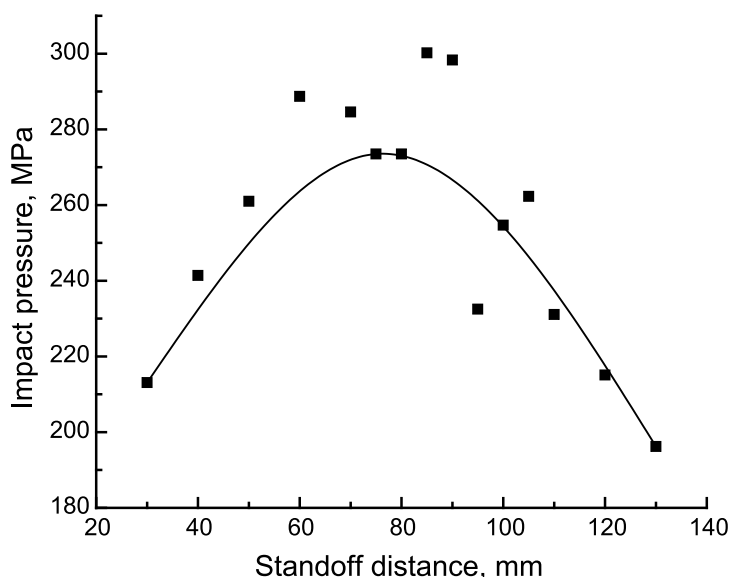

Fig. 16. Relationship between standoff distance and impact pressure of WCP.

clouds along the jet direction.

The main technological parameters of the WCP process include working pressure ( $P, P=0 \mathrm{MPa}$ to $32 \mathrm{MPa})$, nozzle diameter $(D, D=1 \mathrm{~mm})$, standoff distance $(S, S=75 \mathrm{~mm}$ to $90 \mathrm{~mm})$, duration time ( $t$, a variable).

\subsection{Experimental method for estimating impact-pressure distribution}

To obtain the impact pressure distribution on the section with different standoff distances, a novel high-prescale measurement film produced by the FUJI PHOTO FILE Company of Japan was adopted to measure the pressure distribution in this study. The measurement of pressure ranges from $130 \mathrm{MPa}$ to $300 \mathrm{MPa}$. The experiments were performed under the same processing conditions and experimental environment as those in the simulation. The pressure-sensitive film comprises polyester bases, a color-developing layer, and a microencapsulated color-forming layer. The microcapsules are broken when pressure is applied, and the color-forming material reacts with the colordeveloping material. Red patches appear on the film wherever contact pressure is applied. The indicated color density varies according to the different contact pressure levels. The FPD-305 E color indicator and the FPS-307 E software were used to change the color level based on the corresponding pressure value. The schematic of the impact-pressure measuring equipment is shown in Fig. 14. The support of the pressure-sensitive film is installed at the center of the bottom of the vertical-type test water tank. The duration time is $5 \mathrm{~s}$.

\subsection{Experimental results and analysis}

A sample pressure-sensitive film treated by the WCP process is shown in Fig. 15(a). A red, flame-shaped area with uneven concentration was formed when pressure was applied on the pressure-sensitive paper. The FPD-305 E color indicator was used to read quantitative data and to record the discoloration concentration on the pressuresensitive paper. The FPS-307 E software was used to change the color level based on the corresponding pressure value. A standard color swatch of discoloration concentration is shown in Fig. 15(b). The pressure corresponding to the minimum concentration of 0.1 is $130 \mathrm{MPa}$, whereas the maximum concentration of 1.3 corresponds to $300 \mathrm{MPa}$. In addition, a photo of bubble clouds along the cavitation jet direction taken by a high-speed camera is shown in Fig. 15(c). The picture conforms well to the color distribution on the pressure-sensitive film after treatment.

Impact pressures with different standoff distances are shown in Fig. 16. The maximum measured impact pressure induced by the collapse and rebound of a cavitation bubble reaches up to $300 \mathrm{MPa}$ at a standoff distance of $85 \mathrm{~mm}$, indicating a strong processing capability. 


\section{Conclusion}

Both the experimental studies and numerical simulations in this paper were performed to facilitate further understanding of cavitation behavior in the WCP process. FLUENT was used to simulate cavitation behavior both inside and outside the conical nozzle and the convergent-divergent nozzle. The cavitation behavior induced by the convergent-divergent nozzle with different inlet pressures was also investigated. Fujifilm pressure-sensitive paper was used to measure the distribution of the impact pressure induced by the collapse and rebound of a cavitation bubble along the jet direction in the WCP process. The conclusions are as follows:

(1) The RNG $\kappa-\varepsilon$ turbulence model is suitable for simulating the cavitation behavior of the cavitation flow field, including velocity, vapor fraction, and so on, both inside and outside the nozzle in the WCP process.

(2) Different nozzle shapes can have a significant effect on the cavitation phenomenon. A pressure drop of $-20 \mathrm{MPa}$ occurs at the throat of the convergent-divergent nozzle but not in the conical nozzle. This phenomenon promotes the occurrence of cavitation. Moreover, the dropping pressure and the change in the expanding area of the nozzle aggravate the incidence of the cavitation phenomenon.

(3) Different inlet pressure values are also key factors that influence the intensity of the cavitation phenomenon when the outlet pressure remains unchanged. Simulation results show that increasing inlet pressure can enhance the intensity of the cavitation.

(4) Fujifilm pressure-sensitive paper was used to measure the distribution of impact pressure induced by the collapse and rebound of a cavitation bubble along the jet direction in the WCP process. The impact pressure was found to reach up to $300 \mathrm{MPa}$.

(5) The numerical results show that the severe cavitation phenomenon occur in inside and outside of convergentdivergent nozzle when the inlet pressure is $32 \mathrm{MPa}$. The experimental results show that the shock wave pressure induced by the cavitation phenomenon reaches up to $300 \mathrm{MPa}$. From this perspective, the experimentresults and numerical simulation results is consistent.

\section{Acknowledgments}

This research receives ongoing support from the High-Tech Research Center and Open Research Center at the Saitama Institute of Technology, the Doctor Scientific Startup Foundation of Liaoning Province in China (No. 20091035), the Key Laboratory Projects of Educational Department of Liaoning Province in China (No. LS 2010085), the Excellent Talents Projects of Educational Department of Liaoning Province in China (No. LJQ 2011025), and Special Research Foundation of University of Science and Technology Liaoning (No. 2012TD03).

\section{References}

[1] D.Y. Ju, M. Qin and R. Koubayashi, Effect of aeration on superficial residual stress level of carburized and quenched gears treated by water cavitation peening, Surface Engineering 22 (2006), 219-223.

[2] M. Qin, D.Y. Ju and R. Oba, Investigation of the influence of incidence angle on the process capability of water cavitation peening, Surface and Coatings Technology 12(4) (2006), 1409-1413.

[3] H. Soyama, Introduction of compressive residual stress using a cavitating jet in air, Journal of Engineering Materials and Technology 126 (2004), 123-128.

[4] B. Han, D.Y. Ju and W.P. Jia, Influence of water cavitation peening with aeration on fatigue behaviour of SAE1045 steel, Applied Surface Science 253 (2007), 9342-9346.

[5] H. Soyama, M. Shimizu and Y. Hattori, Improving the fatigue strength of the elements of a steel belt for CVT by cavitation shotless peening, Journal of Materials Science 43 (2008), 5028-5030.

[6] H. Soyama and D.O. Macodiyo, Fatigue strength improvement of gears using cavitation shotless peening, Tribology Letters 18(2) (2005), 181-184.

[7] G.L. Chahine, Strong interactions bubble/bubble and bubble/flow, in: Bubble Dynamics and Interface 630 Phenomena, J.R. Blake et al., eds, 1994, pp. 195-206.

[8] M. Deshpande, J. Feng and C.L. Merkle, Numerical modeling of thermodynamic effects of cavitation, AMSE Journal of Fluids Engineering 119 (1997), 420-427.

[9] W. Yuan, J. Sauer and G.H. Schnerr, Modeling and computation of unsteady cavitation flows in injection nozzles, in: 1st International Colloquium on Microhydrodynamics, Paris, France (2000). 
[10] E. Giannadakis, D. Papoulias, M. Gavaises, C. Arcoumanis, C. Soteriou and W. Tang, Evaluation of the predictive capability of Diesel nozzle cavitation models, SAE Paper, No 2007-01-0245.

[11] V. Srinivasan, A.J. Salazar and K. Saito, Modeling the disintegration of cavitating turbulent liquid jets using a novel VOF-CIMD approach, Chemical Engineering Science $\mathbf{6 5}$ (2010), 2782-2796.

[12] J. Li and H.S. Chen, Numerical simulation of micro bubble collapse near solid wall in fluent environment, Tribology 28(4) (2008), 311315.

[13] S.S. Deng, Y.H. Shen and Z.J. Li, Numerical simulation on internal flow field in nozzle with cavitations water jet, Journal of Logistical Engineering University 24(2) (2008), 57-60.

[14] Y.Y. Lu, X.C. Wang and Y. Kang, Simulation of cavitation jet flow in a convergent-divergent nozzle water jet, Journal of China University of Petroleum 33(6) (2009), 57-60.

[15] Z. Qin, K. Bremhorst and H. Alehossein, Simulation of cavitation bubbles in convergent divergent nozzle water jet, Journal of Fluid Mechanics 573 (2007), 1-25.

[16] S.S. Deng, Numerical simulation and experimental study of cavitation jet flow, Fuzzy Info and Eng 2 (2009), 907-913.

[17] D. Choudhury, Introduction to the renormalization group method and turbulence modeling, Fluent Inc Technical Memorandum TM-107, 1993. 

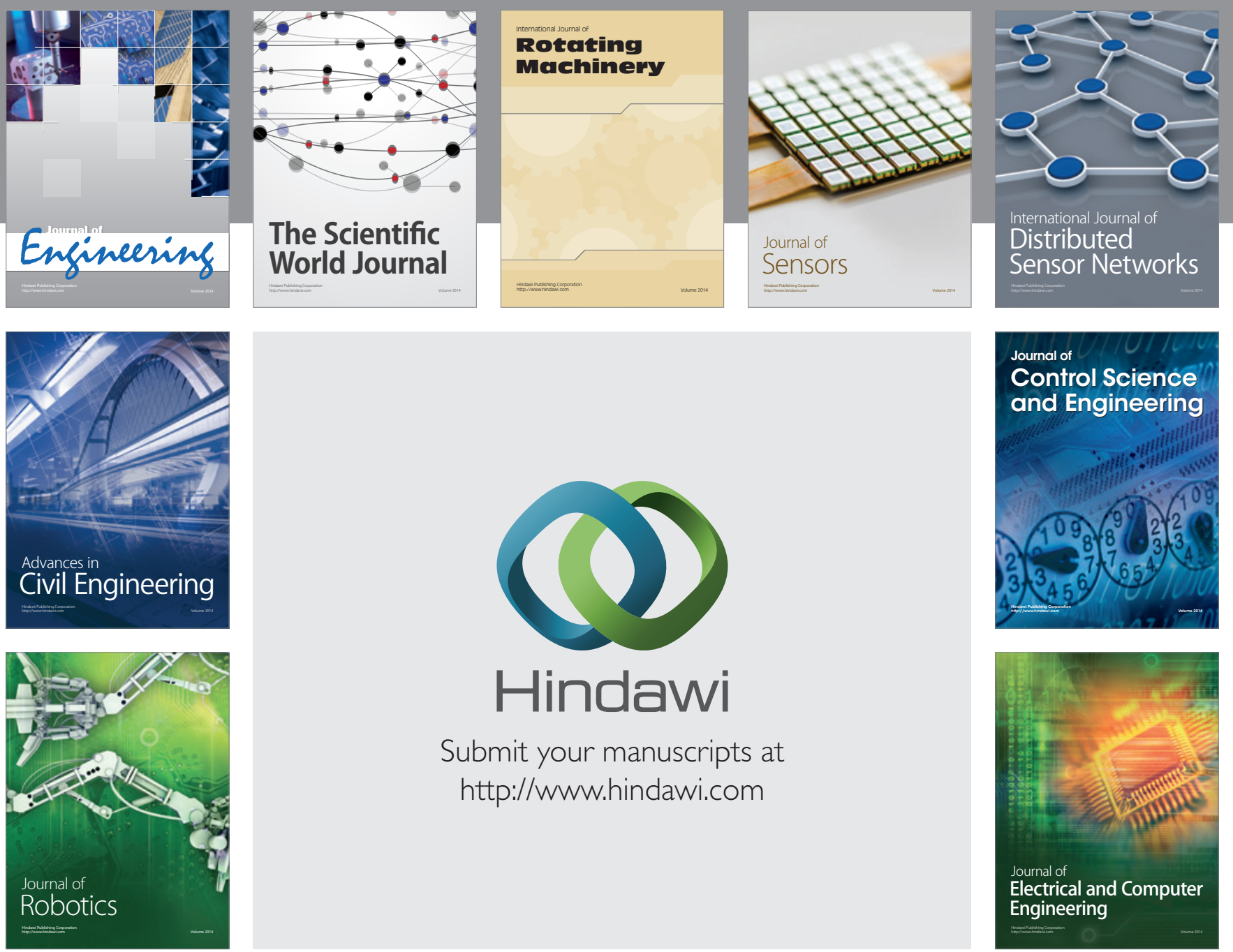

Submit your manuscripts at

http://www.hindawi.com
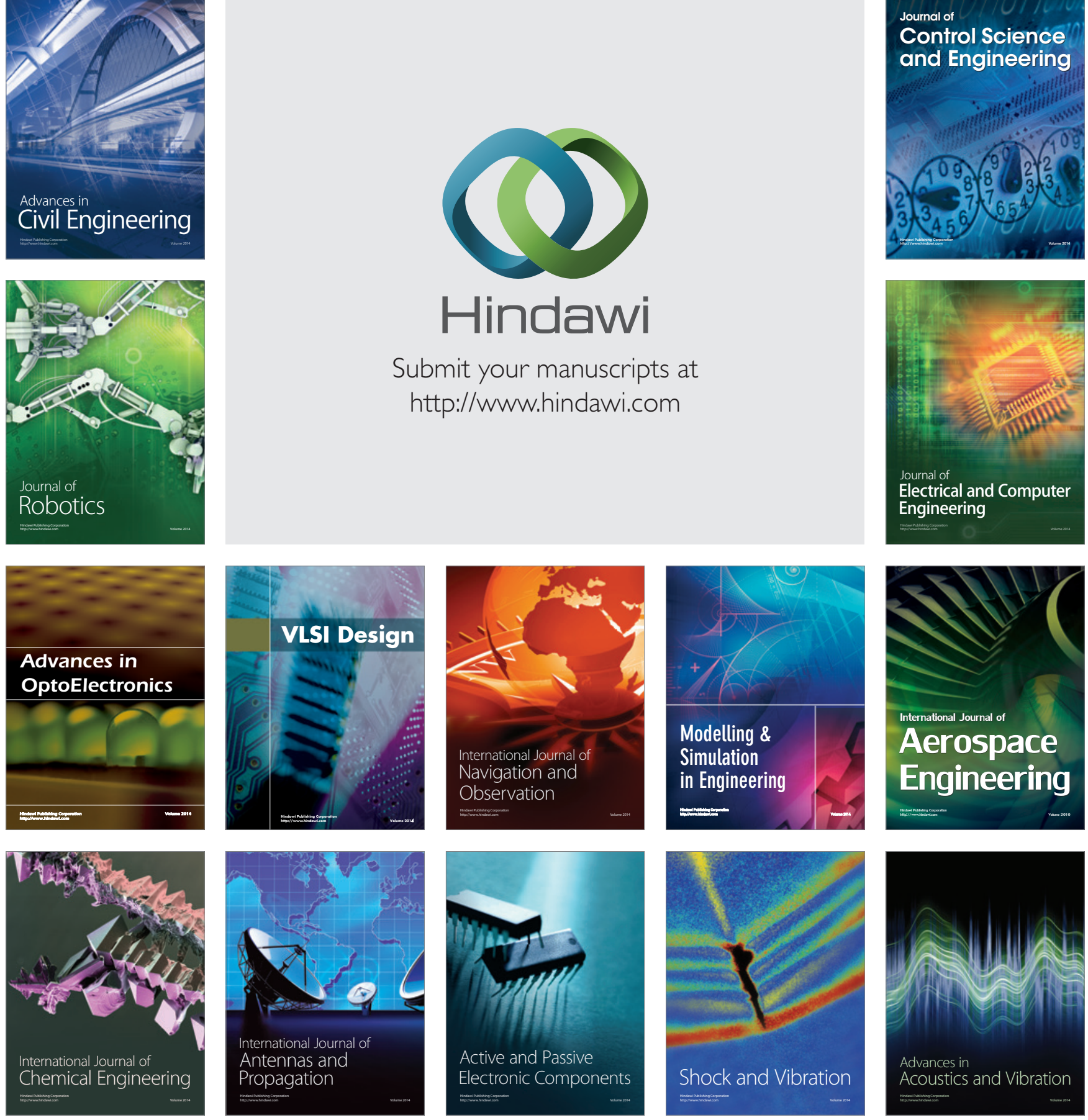